\title{
Numerical study of a control scheme on flow-induced cavity noise
}

\author{
Lulu Liu $^{1,2, *}$, Jin Liu ${ }^{1,2}$, and Shijin Lyu ${ }^{1,2}$ \\ ${ }^{1}$ National Key Laboratory on Ship Vibration \&Noise, Wuxi 214000, China \\ ${ }^{2}$ China Ship Scientific Research Center, Wuxi 214000, China
}

\begin{abstract}
A numerical procedure for flow induced cavity noise is established in the paper. The procedure is based on large eddy simulation and FW-H acoustic analogy. The computational scheme is validated by comparing with experimental data. The change of flow induced noise along with cavity length, cavity depth and velocity is studied. A noise control scheme, which includes upright grille and oblique grille, is designed for reducing the flow-induced cavity noise. It turns out that the oblique grille shows superiority in the reduction of cavity noise by modifying the flow structure of the sheat layer.
\end{abstract}

\section{Introduction}

Although the majority of interest in the cavity flow problem has traditionally come from the aerospace industry, cavity noise can also be a major source of underwater vehicles due to the flow over cavities such as drain holes. It is characterized by a high noise level with a relatively low frequency, ranging from 10 to $50 \mathrm{~Hz}$. Its magnitude may exceed $140 \mathrm{~dB}$. The prediction of flow-induced noise is an important and complex issue in fluid-dynamic acoustics field, which has inspired a great deal of research. Also, the elimination of such noise is becoming of increasing strategic importance.

Various techniques, both active and passive, have been employed to reduce the levels of flow-induced cavity noise. Most of these techniques may be viewed as modifying the upstream or downstream flow regions, or both. Active control methods have the potential to be continuously changed to adapt to different flow conditions. An adaptive feedforward control method was described by Williams[1], in which a linear quadratic regulator was used to control a single mode at low Mach number. The approach reduces a single tone by $15 \mathrm{~dB}$, but couldn't suppress multiple tones. Gareth J. Bennett[2] examined experimentally the use of a fluidic spoiler to suppress the acoustic resonance within a partially closed cylindrical cavity subject to a grazing flow. The result is that the grazing flow can no longer detach from the upstream leading edge of the cavity and thus vortex shedding is suppressed. Often, greater benefits are obtained by using passive techniques. Passive control of cavity oscillations has been successfully implemented via geometric modifications adopting, for example, fixed fences, spoilers, ramps. Sarno and Franke[3] tested a static fence and a pulsating fence at the upstream of the cavity. It turned out that a static fence with a height of 1 boundary layer thickness reduced the level by about $10 \mathrm{~dB}$ at $\mathrm{M}=0.62$. The techniques described above were all tested at Mach numbers mostly greater than 0.2 . The effectiveness of these techniques at lower Mach number has not been demonstrated. Arunajatesan [4] studied modeling and control of Helmholtz resonance in cavities in submarine structures and investigated the effectiveness of leading edge ramps in attenuating the shear layer oscillations over a cavity covered with a slotted plate. It was seen that the ramp height was a function of the length of a cavity opening. Typical height greater than $5 \%$ of the cavity length was observed to be effective.

In this paper, the numerical prediction of flowinduced cavity noise is accomplished using large eddy simulation technique combined with FW-H acoustic analogy method. Then the numerical method is applied to examine the control of the flow-induced noise in the cavity.

\section{Computational methods}

\subsection{Large Eddy Simulation (LES) Model}

Speaking of time-dependent Navier-Stokes computations, LES has the advantage of being less demanding in terms of computational power. This is because only the most energetic scales are resolved and the influence of the smaller scales (or subgrid scales) is modeled in LES. LES is based on two basic assumptions. One is that transport is largely governed by large-scale unsteady flow and these structures can be computationally resolved. And the other is that small-scale flow features can be undertaken by using appropriate subgrid scale turbulence models. In LES, the motion is separated into small and large eddies, this separation is achieved by means of a low-pass filter. The filter function, $G\left(x, x^{\prime}\right)$, implied here is then

\footnotetext{
* Corresponding author: LL300100@aliyun.com
} 


$$
G\left(x, x^{\prime}\right)=\left\{\begin{array}{l}
1 / V, x^{\prime} \in V \\
0, \text { otherwise }
\end{array}\right.
$$

Filtering the Navier-Stokes equations, one obtains

$$
\begin{array}{r}
\frac{\partial \rho}{\partial t}+\frac{\partial}{\partial x_{i}}\left(\rho \bar{u}_{i}\right)=0 \\
\frac{\partial}{\partial t}\left(\rho \bar{u}_{i}\right)+\frac{\partial}{\partial x_{j}}\left(\rho \bar{u}_{i} \bar{u}_{j}\right)= \\
\frac{\partial}{\partial x_{j}}\left(\mu \frac{\partial \sigma_{i j}}{\partial x_{j}}\right)-\frac{\partial \bar{p}}{\partial x_{i}}-\frac{\partial \tau_{i j}}{\partial x_{j}}
\end{array}
$$

where filtered quantities are denoted by an overbar, $\sigma_{i j}$ is the stress tensor due to molecular viscosity and $\tau_{i j}$ is the subgrid-scale stress. The subgrid-scale stresses resulting from the filtering operation are unknown, and require modeling. Dynamic smagorinsky model is used in this paper to simulate subgrid-scale stresses.

\subsection{The FW-H acoustic analogy}

Ffowcs Williams and Hawkings[5] utilized the generalized function theory to obtain the classic equation that has become associated with their names. This is the most general form of Lighthill's acoustic analogy and is appropriate for computing the acoustic field when solid boundaries play a direct role in the generation of sound. The FW-H equation may be written in differential form as

$$
\begin{aligned}
& \frac{1}{c_{0}^{2}} \frac{\partial^{2} p^{\prime}}{\partial t^{2}}-\nabla^{2} p^{\prime}=\frac{\partial^{2}}{\partial x_{i} \partial x_{j}}\left[T_{i j} H(f)\right] \\
& -\frac{\partial}{\partial x_{i}}\left\{\left[P_{i j} n_{j}+\rho u_{i}\left(u_{n}-v_{n}\right)\right] \delta(f)\right\} \\
& +\frac{\partial}{\partial t}\left\{\left[\rho_{0} v_{n}+\rho\left(u_{n}-v_{n}\right)\right] \delta(f)\right\}
\end{aligned}
$$

where $T_{i j}$ is Lighthill stress tensor, $H(f)$ is Heaviside function, where $f=0$ defines the integration surface, $\delta(f)$ is Dirac delta function, $u$ is fluid velocity, $v$ is body surface velocity, $c$ is the ambient speed of sound, $n$ is a normal vector that points into the fluid.

The FW-H approach has several advantages. Firstly, the three source terms on the right-hand side of this equation have clear physical meaning. The first term is the Lighthill stress tensor Tij and represents the generation of sound by the geometry and kinematics of the body. The second term represents the sound generated by the force that acts on the fluid as a result of the presence of the body, and the last term represents the sound generated due to the volume displacement effects of the surface. Secondly, the separation of the source terms is numerically friendly because not all terms must be computed in any circum- stances, especially when a particular source does not contribute to the sound field. Lastly, most FW-H based computer codes are relatively mature and have robust numerical algorithms that have been validated for many aeroacoustic problems of industrial interest.

\section{Geometries and computational settings}

The basic flow configuration consists of grazing flow over cavities. In the present work, we consider cavity models with different lengths and depths, together with various inlet velocities. One representative of cavity geometries investigated here is depicted in Fig.1. The cavity opening is $320 \mathrm{~mm} \times 194 \mathrm{~mm} \times 15 \mathrm{~mm}$. The cavity body size is $360 \mathrm{~mm} \times 215 \mathrm{~mm} \times 785 \mathrm{~mm}$. Four receivers are set up in the computational domain to explore the characteristics of flow-induced cavity noise.

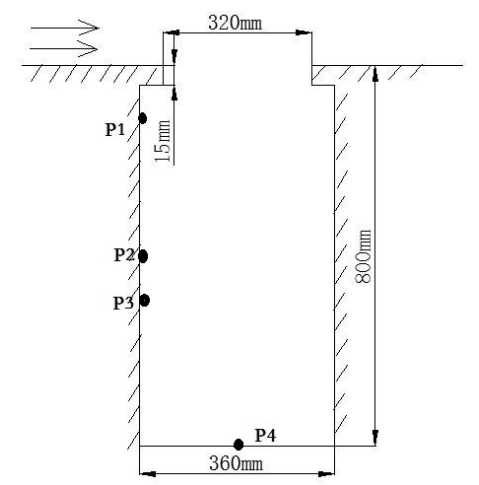

Fig. 1. Cavity geometry.

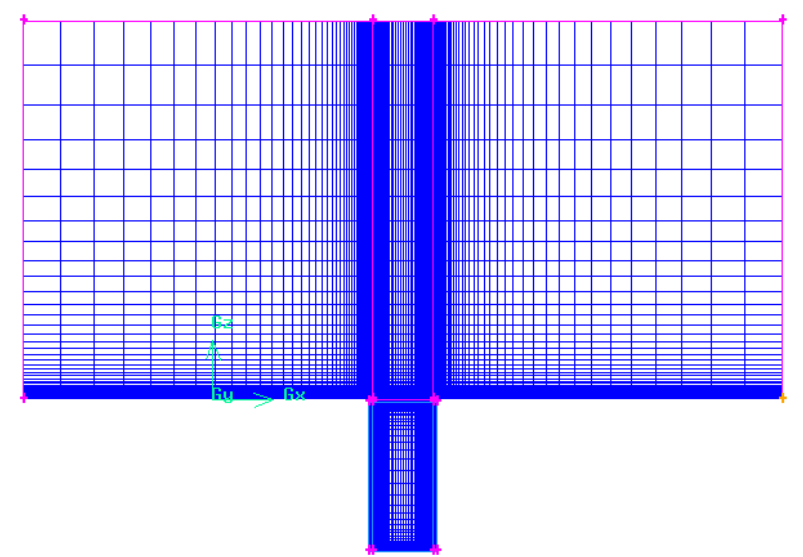

Fig. 2. The computational domain with grids.

The computational domain with meshes is displayed in Fig.2. The differential equations are discretized by finite volume method. The velocity-pressure coupling is based on SIMPLE algorithm. Second order spatial and temporal discretization schemes with fine grids in the immediate region of the cavity are adopted and a small time step is used to capture the unsteady flow physics. The time step is $2 \times 10-4 \mathrm{~s}$.

\section{Results and discussion}

\subsection{Validation of numerical method}

In order to validate the numerical method, an experiment aiming at measuring the flow-induced cavity noise is 
carried out in the cavitation mechanism tunnel of CSSRC. The test section is $845 \mathrm{~mm}$ long and $225 \mathrm{~mm}$ $\times 225 \mathrm{~mm}$ in cross section, which is in accordance with the numerical model. An open-top rectangular box with a span-wise width of $215 \mathrm{~mm}$, length of $360 \mathrm{~mm}$, and depth of $785 \mathrm{~mm}$ is used as the model cavity. There is a rectangular aperture with a span-wise width of $194 \mathrm{~mm}$ and a length of $320 \mathrm{~mm}$ along the centerline on the test section's floor. The cavity center is aligned with the center of the rectangular aperture. This model is referred to as Model A in the following sections. Figure 3 shows a schematic representation of Model A. Four hydrophones are placed at the same position in the cavity as the numerical simulation. Noise characteristics of different locations near the opening and in the cavity are obtained.

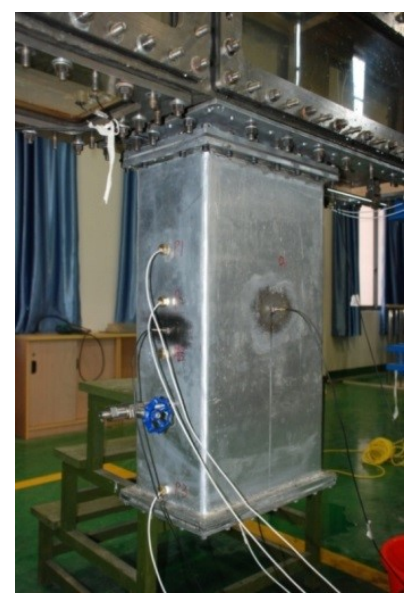

Fig. 3. Experimental set-up of cavity noise measurement.

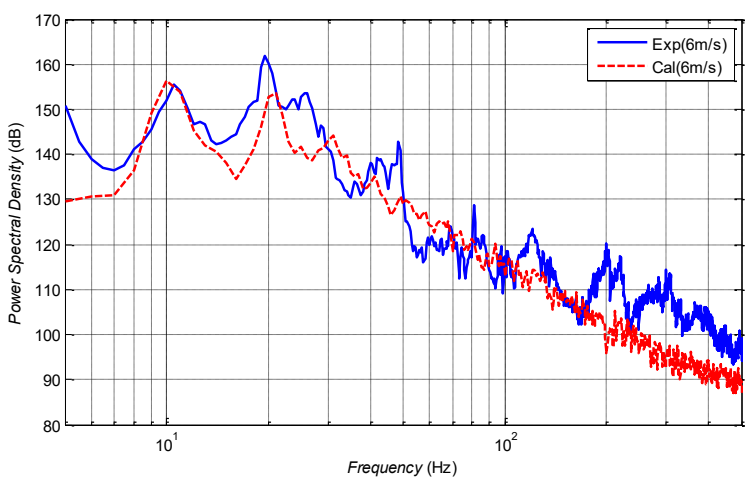

Fig. 4. Comparison of simulated and experimental power spectral density for flow-induced cavity noise.

It's known that flow past an open cavity gives rise to self-sustained oscillations. The preferred modes for the dynamic oscillation are given by the semi-empirical formula of Rossiter[6].

$$
f_{n}=\frac{n-0.25}{M+1 / 0.57} \times \frac{U_{0}}{L}
$$

where $f_{n}$ is the modal oscillation frequency, $n$ is the modal number, $U_{0}$ is the inflow velocity, $M$ is the Mach number and $L$ is the cavity opening length. Fig.4 presents the power spectral density at monitoring point (P1), where the water velocity is $6 \mathrm{~m} / \mathrm{s}$. Comparisons between numerical and experimental data demonstrate good agreement in both the frequency and amplitude of the oscillations within the cavity, as is shown in Fig. 4.
Table.1 lists the experimental, computational and theoretical results of the shear oscillation with a cavity opening length of $320 \mathrm{~mm}$. The calculated shear oscillation frequency is basically the same as the test result, and the total sound level deviation is less than 4 $\mathrm{dB}$. It is obvious that the numerical method developed in this paper is reliable, and can be used to analyze the flow-induced cavity noise. It also shows that Rossiter's formula underestimates the modal frequency of cavity shear layer oscillation.

Table 1. Comparison of simulated, experimental and heoretical shear oscillation frequency.

\begin{tabular}{|c|c|c|c|}
\hline & & $\mathrm{n}=1$ & $\mathrm{n}=2$ \\
\hline \multirow{3}{*}{$\begin{array}{c}320 \mathrm{~mm} \\
(6 \mathrm{~m} / \mathrm{s})\end{array}$} & Experimental result & 10.5 & 19.5 \\
\cline { 2 - 4 } & Calculation result & 10 & 21 \\
\cline { 2 - 4 } & Theoretical result & 8.0 & 18.7 \\
\hline
\end{tabular}

\subsection{Characteristic analysis of flow-induced cavity noise}

The paper takes a move to perceive the effect of cavity size and velocity on flow-induced noise. Firstly, to analyze the impact of cavity depth on power spectra of cavity noise, the depth is set to $400 \mathrm{~mm}, 800 \mathrm{~mm}$, $1200 \mathrm{~mm}$ respectively. For a cavity with an opening length of $50 \mathrm{~mm}$, comparison of sound power spectral density with different cavity depth is presented in Fig.5. With cavity depth increasing from $400 \mathrm{~mm}$ to $800 \mathrm{~mm}$, the shear oscillation frequency of the first two modes remains unchanged while the amplitude of the first mode drops by about $10 \mathrm{~dB}$. With cavity depth continuing increasing to $1200 \mathrm{~mm}$, both the shear oscillation frequency and peak value no longer alter. Thus the cavity depth is set to $800 \mathrm{~mm}$ in the following sections.

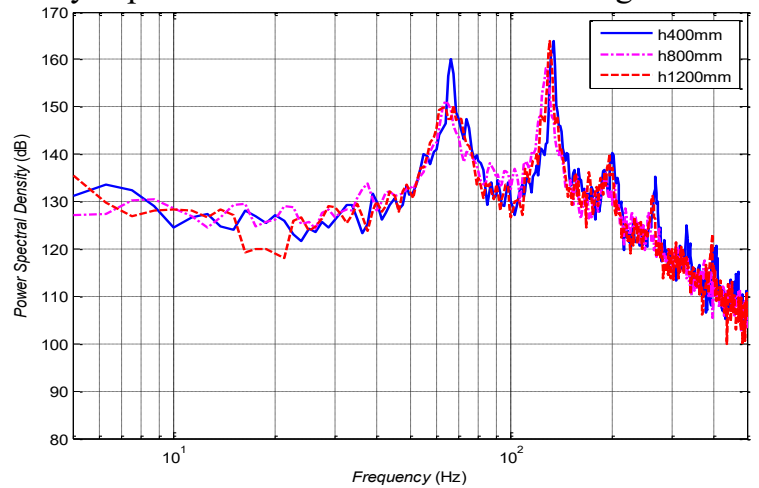

Fig. 5. Effect of cavity depth on flow-induced cavity noise.

The present study also investigates the impact of cavity length on flow-induced noise. Five cavity lengths $(\mathrm{L}=20 \mathrm{~mm}, 50 \mathrm{~mm}, 100 \mathrm{~mm}, 150 \mathrm{~mm}, 200 \mathrm{~mm}, 320 \mathrm{~mm})$ are considered respectively in the case of inflow velocity $=6 \mathrm{~m} / \mathrm{s}$. Results clearly indicate that both the modal frequency and amplitude of shear oscillation are sensitively dependent on the cavity length, as is seen in Fig.6. The modal frequency moves to the lower frequency band as the cavity length increases. While the opening size grows from $20 \mathrm{~mm}$ to $320 \mathrm{~mm}$, the corresponding firstorder shear oscillation frequency shifts from $140 \mathrm{~Hz}$ to $11 \mathrm{~Hz}$, and the second-order shear 
oscillation frequency decreases from $281 \mathrm{~Hz}$ to $21 \mathrm{~Hz}$. It can be concluded that the larger the cavity length, the lower the shear oscillation frequency of the cavity.

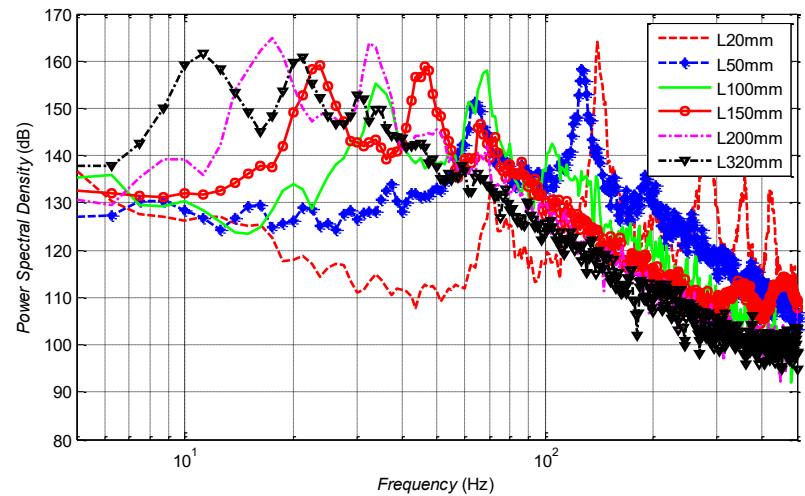

Fig. 6. Effect of cavity streamwise length on flow-induced cavity noise.

Additional simulations are subsequently performed to assess the influence of freestream velocity on the nature of the cavity oscillations. Three freestream speeds have been examined $\left(U_{0}=4.5 \mathrm{~m} / \mathrm{s}, 6 \mathrm{~m} / \mathrm{s}, 9 \mathrm{~m} / \mathrm{s}\right)$ where the cavity length is $320 \mathrm{~mm}$. It is evident from Fig.7 that the peak frequency of fluid dynamic oscillation goes up as the increase of inlet velocity. Besides, the total sound pressure level in the frequency range $5 \mathrm{~Hz} \sim 500 \mathrm{~Hz}$ is $166 \mathrm{~dB}, 170 \mathrm{~dB}$ and $175 \mathrm{~dB}$ at different freestream conditions. That is, when the inlet velocity doubles, the total sound pressure level is increased by about $9 \mathrm{~dB}$.

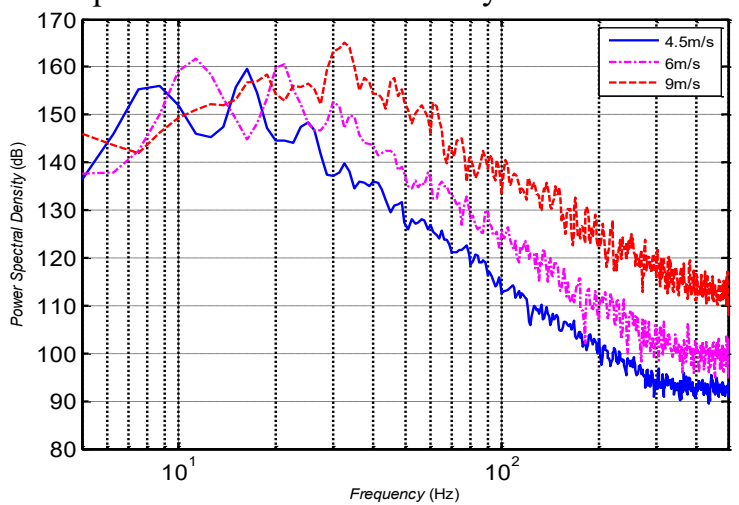

Fig. 7. Effect of inflow velocity on flow-induced cavity noise.

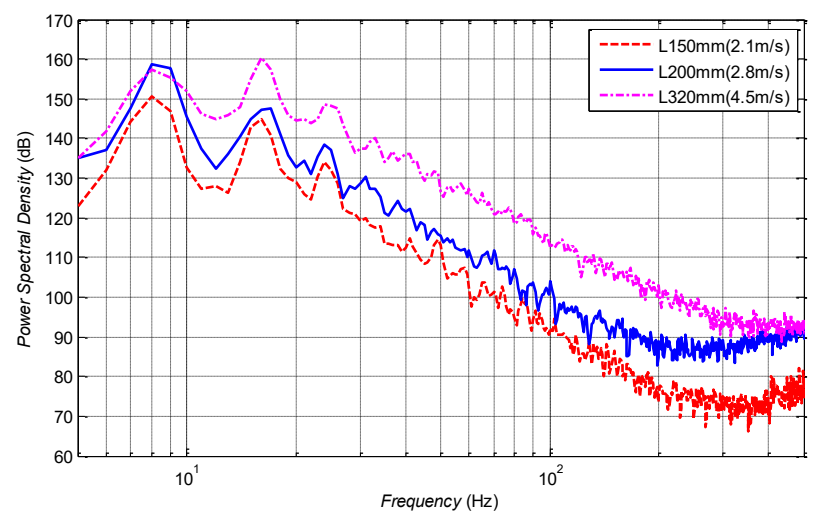

Fig. 8. Comparison of power spectral density for flow-induced cavity when $U_{0} / L=$ constant.

Fig.8 demonstrates the predicted results of power spectral density of cavity noise at $L=150 \mathrm{~mm}, 200 \mathrm{~mm}$, and $320 \mathrm{~mm}$ when $U_{0}=2.1 \mathrm{~m} / \mathrm{s}, 2.8 \mathrm{~m} / \mathrm{s}$ and $4.5 \mathrm{~m} / \mathrm{s}$, sep- arately. It is clear that $U_{0} / L$ is a fixed value and the peak frequency of shear oscillation coincides in all cases. This phenomenon is also consistent with the empirical formula of Rossiter modes. Moreover, the peak value increases with the increase of velocity, so does the total sound pressure level.

\subsection{Control scheme on flow-induced cavity noise}

Adding grille to the cavity may alter the flow field and phase relations, which in turn results in the change of acoustic field. In this study, with the aid of the numerical tool described earlier, two types of grille are developed for reducing the flow-induced cavity noise. Unlike traditional slotted plate covering the cavity, an upright grille which consists of two parts at right angle is designed here at the beginning. It shows that, however, the usage of upright grille may cause unsteady oscillations at other frequencies. Then an improved design should be sufficient to reduce the cavity noise at all frequencies by introducing an oblique grille. Fig.9a and $9 \mathrm{~b}$ show the schematic representations of these two designs for a cavity length of $320 \mathrm{~mm}$, which are referred to as upright grille and oblique grille, respectively.

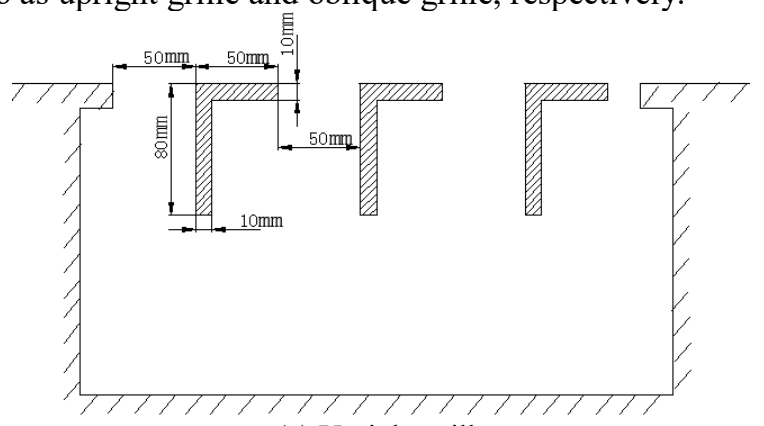

(a) Upright grille

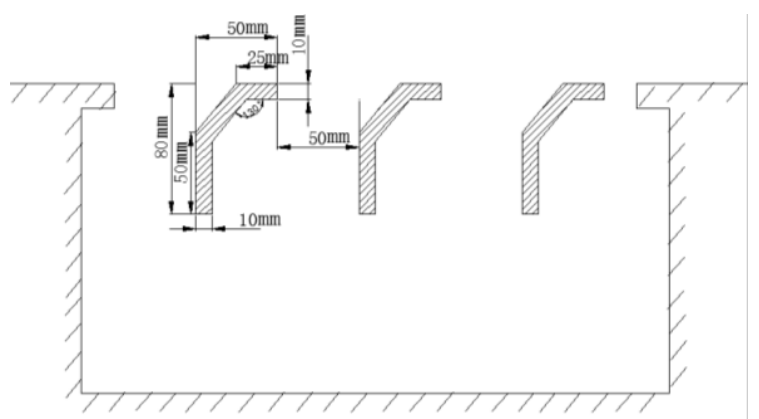

(b) oblique grille

Fig. 9. Two models : upright grille and oblique grille.

To provide clear evidence of the effectiveness of grille for noise suppression, the comparison of power spectral density between different models is depicted in Fig.10. It can be observed that the total sound pressure level of upright grille and oblique grille in the frequency range $5 \sim 500 \mathrm{~Hz}$ is $170 \mathrm{~dB}$ and $161 \mathrm{~dB}$ respectively, in comparison with a total sound pressure level of $152 \mathrm{~dB}$ for the original model $\mathrm{A}$. Note that both upright grille and oblique grille demonstrate significant reduction in the sound pressure level in the frequency range $(<50 \mathrm{~Hz})$. In the case of upright grille, the first-order shear oscillation frequency shifts from $11 \mathrm{~Hz}$ to $66 \mathrm{~Hz}$, while 
the second-order shear oscillation frequency increases from $21 \mathrm{~Hz}$ to $124 \mathrm{~Hz}$, together with a dramatic decline of peak value. Contrastingly, the power spectral density curve of the cavity is relatively smooth with no peaks in the case of oblique grille. Thus the oblique grille presents a much more favorable attenuation of flowinduced cavity noise.

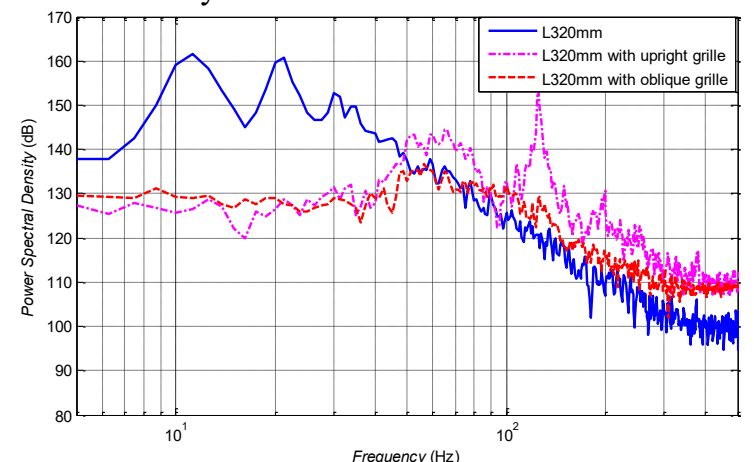

Fig. 10. Effect of grille on power spectral density of $320 \mathrm{~mm}$ long cavity.

Simulation results of upright grille and oblique grille are also compared to the case of cavity with smaller opening $(\mathrm{L}=50 \mathrm{~mm})$ as illustrated by the Fig. 11 . Upright grille and $50 \mathrm{~mm}$ long cavity share the same peak frequency, while the peak value for upright grille is slightly lower than the small cavity. However, oblique grille helps eliminate the peaks of the power spectrum curve. It suggests that for large openings on underwater vehicles, the grille is equivalent to changing a large opening into a number of small openings with grille spacing. Therefore, the control scheme of upright or oblique grille can be adopted to control the flow excited noise where the opening size is not adjustable.

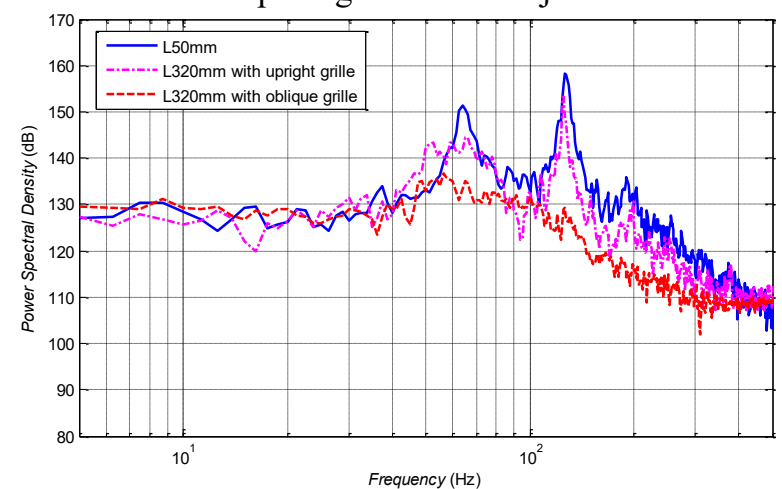

Fig. 11. Comparison of power spectral density between grilled $320 \mathrm{~mm}$ long cavity and $50 \mathrm{~mm}$ long cavity.

\section{Conclusions}

A computational scheme based on large eddy simulation and FW-H acoustic analogy is developed in the paper. Validated by comparison with experimental data, it allows a reasonable prediction of modal frequencies of shear oscillation for flow past a cavity. The change of flow induced noise along with cavity length, cavity depth and velocity is studied. Results clearly indicate that both the modal frequency and amplitude of shear oscillation are sensitively dependent on the cavity length and inflow velocity. More importantly, the present work concentrates on a control scheme of two designs, which involves installing upright and oblique grille in the cavity opening. The effect of grille on sound field is analyzed. It indicates that the oblique grille shows superiority in the reduction of the cavity noise, concerning the fact that it helps flatten the sound power spectrum curve and the total sound pressure level decreases by about $17 \mathrm{~dB}$. Future study should be directed to a better understanding of the grille for reduction of the flow-induced noise in cavities

\section{References}

1. Williams, D.R. and Morrow, J., 31st AIAA Fluid Dynamics Conference, No. AIAA-2001-2769.

2. Gareth J. Bennett, 22ndAIAA/CEAS Aeroacoustics Conference. No. AIAA-2016-2756.

3. Sarno, R.L. and Franke, M.E. AIAA Paper No. 90-4018-CP (1990)

4. S. Arunajatesan, $14^{\text {th }}$ AIAA/CEAS Aeroacoustics Conference. No. AIAA-2008-2850.

5. J. E. Ffowcs Williams and D. L. Hawkins, Proc. Roy. Soc. London, 264 A, 321-342.

6. J. E. Rossiter, Aeronautical Research Council Reports and Memo No. 3438 (1964)

7. D. Rockwell, E. Naudascher, Journal of Fluids Engineering, 100, 152-165 (1978)

8. Wang et al., Acta Acustica United with Acustica, 98, 600-610 (2012) 